\title{
A rat model for studying neural stem cell transplantation
}

\author{
Xue-mei ZHOU ${ }^{1, \#}$, Jing-bo SUN ${ }^{1, \#}$, Hui-ping YUAN ${ }^{1, \#, *}$, Dong-lai WU ${ }^{2}$, Xin-rong ZHOU ${ }^{1}$, Da-wei SUN ${ }^{1}$, Hong-yi LI ${ }^{1}$, Zheng-bo
} $\mathrm{SHAO}^{1}$, Zhi-ren ZHANG ${ }^{3}$

${ }^{1}$ Department of Ophthalmology, Second Affiliated Hospital of Harbin Medical University, Harbin 150086, China; ${ }^{2}$ Harbin Veterinary Research Institute, China Agricultural Academy of Science, Harbin 150001, China; ${ }^{3}$ Key Laboratories of Education Ministry for Myocardial Ischemia and Treatment; Department of Clinical Pharmacy and Cardiology, Second Affiliated Hospital of Harbin Medical University, Harbin 150086, China

\begin{abstract}
Aim: The goal of this project was to develop a rat model for neural stem cell (NSC) transplantation studies in which NSCs were modified with brain-derived neurotrophic factor (BDNF) genes that may permit extensive and reliable analysis of the transplants.

Methods: NSCs were cultured and purified by limiting dilution assay in vitro and infected with recombinant retrovirus pLXSN-BDNF (BDNF-NSCs) and retrovirus pLXSN (p-NSCs). The expression of BDNF genes in transgenic and control NSC groups was measured by FQ-PCR and ELISA assays. NSCs were then transplanted into the subretinal space of normal rat retinas in four groups, which included NSCs alone, BDNF-NSCs, phosphate buffered saline (PBS) control, and normal control. Survival, migration, and differentiation of donor cells in host retinas were observed with optical coherence tomography (OCT), Heidelberg retina angiograph (HRA), and immunohistochemistry, respectively.

Results: The results obtained by FQ-PCR demonstrated that the copy numbers of BDNF gene templates from BDNF-NSCs were the highest among the four groups $(P<0.05)$. Consistent with the results of FQ-PCR, BDNF protein level from the supernatant of the BDNFNSCs group was much higher than that of the other two groups $(P<0.05)$ as suggested by the ELISA assays. HRA and OCT showed that graft cells could successfully survive. Immunohistochemical analysis revealed that transplanted BDNF-NSCs could migrate in the host retinas and differentiate into glial cells and neurons three months after transplantation.
\end{abstract}

Conclusion: BDNF promotes NSCs to migrate and differentiate into neural cells in the normal host retinas.

Keywords: neural stem cells; differentiation; gene therapy; subretinal space transplantation; migration; purification

Acta Pharmacologica Sinica (2009) 30: 1496-1504; doi: 10.1038/aps.2009.151

\section{Introduction}

Neural stem cells (NSCs), which have self-renewal ability and multi-differentiating potential, have been used for treating degenerative diseases and repairing injuries to the central nervous system $^{[1,2]}$. In recent decades, procedures to replace aged or diseased retinal cells with healthy retinal cell allografts or xenografts have been extensively investigated using many models ${ }^{[3-5]}$. Encouraging results suggest that some blindness disorders may be amenable to treatment by cell transplantation. However, the therapeutic efficacy of these mature retinal cells is limited in that they have weak migration capacities in

\footnotetext{
"The three authors contributed equally to this work.

*To whom correspondence should be addressed.

E-mail yuanhp@yahoo.com

Received 2009-05-16 Accepted 2009-09-14
}

vivo. By contrast, some reports show that transplanted NSCs have the advantage to not only differentiate into the host cells, but to also migrate widely and automatically trace the focus of infection $^{[6-8]}$. Unfortunately, NSC engineering and transplantation remain a difficult strategy because of the low rate of success. To this end, we adapted an effective method to purify and modify embryonic NSCs and developed an improved method to transplant the NSCs. These data could provide a reliable experimental model and ultimately provide information for therapeutic intervention.

In general, a large number of purified NSCs are needed to study biological characteristics of NSCs and for use in clinical research; however, the number of NSCs in rat nervous tissue is low, and the cells cannot be sorted by immunomagnetic beads or flow cytometry owing to a lack of specific membrane surface markers. The preparation of a considerable amount of purified NSCs was a handicap for the research and clinical 
application of NSCs. Furthermore, the transplanted NSCs in the host usually differentiated to glial cells instead of neurons in vivo, thereby limiting their therapeutic effects. It has been reported that BDNF promotes NSCs to differentiate into neurons in vitro ${ }^{[9]}$; however, under normal culturing conditions, the amount of BDNF in NSCs is very low, and it dramatically decreases during NSC differentiation as reported by other investigators ${ }^{[10,11]}$. Therefore, it appears that stably enforcing the expression of BDNF in NSCs may facilitate their neuronic differentiation in vivo.

In this study, we used the limiting dilution assay to purify the NSCs from the subependymal zones of embryonic rat. BDNF was overexpressed in these cells by recombinant retrovirus, and the cells were labeled with AAV-EGFP to serve as donor cells for transplantation. The effects of BDNF on NSC migration and neuronic differentiation after transplantation were evaluated. Our results demonstrate that BDNF could promote NSCs to migrate and differentiate into neural cells in the normal host retinas.

\section{Materials and methods \\ Cell culture}

The clean (CL) animals [certificate number: SCXK (Heilongjiang) 2006-010] were obtained from the Animal Center of Harbin Medical University. All procedures in the animal experiments followed the instructions for the care and use of animals provided by the institution.

Adult female Sprague-Dawley (SD) rats at 10-12 weeks of age weighing 220-250 $\mathrm{g}$ at 14.5 days gestation were sacrificed after being anesthetized, and embryonic brains were removed and put in $4{ }^{\circ} \mathrm{C}$ phosphate buffered saline (PBS) followed by removal of overlying meninges and blood vessels. Subependymal zones from the lateral wall of the lateral ventricle were aseptically removed under a stereomicroscope (Olympus, SZ61, Japan). The dissected tissue was transferred to PBS and washed twice, then transferred to Petri dishes, dissociated mechanically, and resuspended in PBS solution before filtrating through a 200-mesh cell sieve to eliminate the debris. The cell suspension was washed in PBS three times to rinse off excess blood and centrifuged at $250 \mathrm{~g} / \mathrm{min}$ for $5 \mathrm{~min}$, and the cell pellet was resuspended in proliferation stem cell medium containing serum-free Dulbecco's modified Eagle's medium (DMEM)/F12 (1:1) supplemented with penicillin $\left(1 \times 10^{5} \mathrm{U} / \mathrm{L}\right)$, streptomycin $\left(1 \times 10^{5} \mathrm{U} / \mathrm{L}\right)$, epidermal growth factor (EGF, 20 $\mathrm{mg} / \mathrm{L}$ ), basic fibroblast growth factor (bFGF, $20 \mathrm{mg} / \mathrm{L}$ ), and synthetic mixture B27 (2\%) (Invitrogen, USA). Viable cells were counted by trypan blue exclusion assay with a hemocytometer. Cells were seeded in T-25 culture flasks at a density of $1 \times 10^{9}$ cells/ $\mathrm{L}$ and were maintained at $37^{\circ} \mathrm{C}$ in an incubator with $5 \% \mathrm{CO}_{2}$. Half of the medium was changed every $4 \mathrm{~d}$, and neurospheres were passaged (1:2 or 1:3) by dissociation through a fire-polished Pasteur pipette into single suspension cells with trypsin-ethylene diamine tetraacetic acid (EDTA) $(0.05 \%-0.02 \%)$ in PBS every 6 to $7 \mathrm{~d}$ (consecutive passage assay).

\section{Limiting dilution assay}

To obtain a more unified cell population, a limiting dilution assay was performed as described previously ${ }^{[12,13]}$. After primary sphere formation was noted, neurospheres were dissociated as described above, and cell numbers were adjusted to give a starting concentration of $1 \times 10^{7}$ cells $/ \mathrm{L}$, from which serial dilutions were made. Final cell dilutions, ranging from 100 cells/well to 1 cell/well, were plated in 96-well microwell plates (Corning Costar, USA) with $0.2 \mathrm{~mL}$ growth medium in each well. After the first two weeks, half of the media was changed. Each well was manually screened for colonies using phase-contrast microscopy (Leica, DMIRE-II, Germany), and only wells that originally contained one single cell were referred to as clones. Secondary clonal neurospheres were collected after incubation for 4 to 6 weeks and seeded in $25-\mathrm{cm}^{2}$ culture flasks to an expanded culture.

\section{Immunohistochemistry}

The primary and two groups of purified neurospheres (consecutive passage assay and limiting dilution assay) were plated in differentiation-promoting condition media [growth factor withdrawal and $10 \%$ fetal bovine serum (FBS, PAA Clone, USA)] on poly-L-lysine $(0.1 \mathrm{~g} / \mathrm{L})$ coated glass coverslips in 12-well plates (Corning Costar, USA) and grown for $7 \mathrm{~d}$. Nestin identification for some of the neurospheres was completed $2 \mathrm{~h}$ after adherence. Cells were fixed with $4 \%$ paraformaldehyde (PA) at $4{ }^{\circ} \mathrm{C}$ for $30 \mathrm{~min}$. Nonspecific binding sites were blocked with $5 \%$ goat serum in PBS, and the samples were permeabilized with $0.1 \%$ Triton X-100 in PBS for $1 \mathrm{~h}$ at room temperature. The specimens were incubated overnight at $4{ }^{\circ} \mathrm{C}$ with the primary antibodies at the following dilutions: mouse anti-nestin 1:100 (BD Pharmingen, USA); mouse anti-NSE 1:10 (Chemicon, USA); rabbit anti-GFAP 1:100 (Boster, China). The secondary FITC-conjugated antibodies were diluted 1:100 (goat anti-mouse or rabbit, ZhongShan GoldenBridge, China) and incubated with cells for $1 \mathrm{~h}$ at $37^{\circ} \mathrm{C}$ protected from light. All antibody dilutions and washes were performed with 0.01 mol/L PBS solution. After the last wash, the samples were briefly rinsed with distilled water and observed using a fluorescence microscope (Leica, DMR, Germany). The nestinpositive cell numbers in dark fields and total cell numbers in bright fields were recorded and then nestin-positive rates were calculated as follows: nestin-positive cell numbers/total cell numbers $\times 100 \%$.

\section{Infection}

On the day prior to infection, purified NSCs from the tertiary generation of limiting dilution assay were finely dissociated and seeded into 24-well plates (Corning Costar, USA) at a density of $1 \times 10^{7} / \mathrm{L}$, then infected with the supernatant of the replication-defective recombinant retrovirus ( $\mathrm{pLXSN-BDNF)}$ containing BDNF genes of rats in the highest titer $\left(3.4 \times 10^{5}\right.$ CFU/cell, constructed by members of our experimental group $^{[14]}$ ) in the presence of $8 \mathrm{mg} / \mathrm{L}$ polybrene (Sigma-Aldrich, USA). After overnight incubation, infected NSCs were 
washed twice with medium and cultured with fresh proliferation stem medium for $2 \mathrm{~d}$ before adding $400 \mathrm{mg} / \mathrm{L}$ antibiotic G418 (Sigma-Aldrich, USA) for selection and maintenance of infected NSCs for the following 2 to 3 weeks (BDNF-NSCs). NSCs were infected with a blank retrovirus (pLXSN) using the same method as described above (p-NSCs). Parallel control NSCs (control-NSCs) were also prepared and underwent the same treatment except for infection and G418 selection.

\section{GFP labeling}

The G418-resistant BDNF-NSCs and p-NSCs, and controlNSCs (cultured for $12 \mathrm{~h}$ before infection) were washed twice with DMEM/F12 followed by infection with the solution of rAAV2/EGFP (adeno-associated viruses type-2/enhanced green fluorescent protein) at multiplicity of infection (MOI) of $1 \times 10^{5}$ (vector genome) vg/cell. After incubation for $1 \mathrm{~h}$, the virus liquid was gently removed from NSCs by centrifuge at $1000 \mathrm{r} / \mathrm{min}$. Then fresh proliferation stem medium was added into a centrifuge tube, and the NSCs were seeded onto T25 flasks and placed into an incubator. On the next day, expression of EGFP were detected by fluorescence microscopy and fluorescence-activated flow cytometry (FACSCalibur, Becton Dickinson, USA).

\section{Real-time fluorescent quantitative PCR assay (FQ-PCR)}

Prior to FQ-PCR assays, the standard curve was constructed from recombinant plasmid pLXSN-BDNF in a series of dilutions from $10^{7}-10^{2}$ copies $/ \mu \mathrm{L}$. An RNeasy Mini Kit (Qiagen, Germany) was used to extract total RNA from the three groups, BDNF-NSCs, p-NSCs, and control-NSCs at the same cell density $\left(5 \times 10^{4}\right)$. The sequences of BDNF primers and the probe are as follows:

BDNF-sense: 5'-GGCCCAACGAAGAAAACCAT-3'; BDNF-antisense: 5'-GAGGCTCCAAAGGCACTTGA-3'; BDNF-probe: FAM-CACTTCCCGGGTGATGCTCAGCATAMRA.

A total of $20-\mu \mathrm{L}$ reaction liquid was added to tubes using a One-Step RT-PCR kit (Invitrogen, USA), according to the manufacturer's instructions. FQ-PCR reactions were performed using an FQ-PCR System (Roter-gene 3000, Corbett Life Science, Australia) under the following conditions: $50{ }^{\circ} \mathrm{C}$ for 15 min, $95^{\circ} \mathrm{C}$ for $2 \mathrm{~min}$, and 40 cycles of $95^{\circ} \mathrm{C}$ for $15 \mathrm{~s}$ plus $60^{\circ} \mathrm{C}$ for $30 \mathrm{~s}$. The target amount was determined by the appropriate standard curve, and these findings were repeated in two separate sets of assays.

\section{Enzyme linked immunosorbent assay (ELISA)}

Cells from BDNF-NSCs, p-NSCs, and control-NSC groups were seeded separately in 24-well plates at a density of $2 \times$ $10^{7}$ cells/L. Neurotrophic factor production of BDNF released by three groups in vitro was measured continually for $4 \mathrm{~d}$ using an ELISA kit (Boster, China) according to the manufacturer's instructions. Each specimen underwent detection three times, and average numbers of OD were obtained using an ultraviolet spectrophotometer (Benchmark PLUS, Bio-Rad, USA).

\section{Subretinal transplantation}

Control-NSCs and BDNF-NSCs were centrifuged, resuspended in $200 \mu \mathrm{L}$ PBS (30000 cells $/ \mu \mathrm{L}$ ), and placed onto ice until transplantation. In this study, the normal female SD rats at 8 weeks of age weighing $0.2-0.25 \mathrm{~kg}$ were deeply anesthetized with an intraperitoneal injection of $10 \%$ chloral hydrate $(0.3 \mathrm{~L} / 100 \mathrm{~kg})$. The pupils were dilated with topical 1\% tropicamide to view the fundus for transplantation surgery for the following four groups: BDNF-NSCs $(n=35)$, control-NSCs $(n=33)$, PBS control $(n=8)$, and normal controls $(n=8$, no surgery). The rats were labeled with numbers and were selected at random on a computer into four groups. Under transpupillary observation with the aid of a 90D preset lens (Ocular Instruments, USA) in the surgeon's left hand, and using a binocular ophthalmic surgical microscope (Zeiss, Germany), a $10 \mu \mathrm{L}$ cell suspension was injected at the equator through the pars plana of the sclera and choroid backwards into the subretinal space of the eye with a $1 \mathrm{~mL}$ syringe and a 29-gauge needle, as described previously ${ }^{[8,9]}$ with a few modifications. The operations were done carefully to avoid damaging the lens, retina, iris, and vessels. The appearance of a domeshaped retinal detachment indicated that the cell suspension was delivered properly into the target subretinal space. After injection, the needle remained within the subretinal space for 2 to 3 min before being slowly withdrawn to prevent reflux of the transplanted cells into the vitreous. The same volume of PBS was injected in the blank controls.

\section{Observation in vivo}

Anesthesia and dilation of pupils of the experimental rats were performed as described above on day 3, 10, 15 and then once per month for 3 consecutive months after transplantation. Survival and migration of each transplanted cell in the subretinas of rats were observed using noninvasive methods, including a Heidelberg retina angiograph (HRA, Heidelberg, Germany) and optical coherence tomography (OCT, Zeiss, Germany) to review migration of donor cells under the subretina and reattachment of retinal detachment of surgical rats, respectively.

\section{Immunohistochemistry}

The rats from two cell transplantation groups $(n=8$ or 9 for each group at four timepoints) and two control groups ( $n=2$ for each group at every time point) were used for immunohistochemistry at day 10 and then monthly for 3 months after transplantation. The rats were given an overdose of anesthesia, and the chests were opened for transcardial perfusion with saline followed by $4 \%$ paraformaldehyde in $0.1 \mathrm{~mol} / \mathrm{L}$ phosphate buffer $(\mathrm{PB})$ until the rats' tails became hard or the eyes became white. To perform immunohistochemistry, the eyes were opened at the corneas after removal from the fossa orbitalis, immersed in $10 \%$ neutral formalin, and postfixed at room temperature for $48 \mathrm{~h}$. The anterior segments and the lenses were cut away, and the resultant eyecups were washed with tap water followed by dehydration through a graded ethanol series. The eyecups were then embedded in paraffin. 
Paraffin sections $(2 \mu \mathrm{m})$ were obtained with an ultramicrotome (Leica, Germany) and attached to glass slides. The sections (a small portion of sections were dedicated to hematoxylin and eosin (HE) staining) were then incubated overnight at 4 ${ }^{\circ} \mathrm{C}$ with primary antibodies: nestin (1:200), NSE (1:100), and GFAP (1:300). After reaction with the primary antibodies, the specimens were washed 3 times with PBS for 5 min and incubated with the corresponding secondary antibodies (horseradish peroxidase (HRP) conjugated goat anti-mouse/rabbit IgG) for $20 \mathrm{~min}$ in a humid box in the dark at room temperature. Coloration was made with 3, 3'-diaminobenzidine (DAB) solution (positive product was labeled brown). Slides were rinsed completely, and nuclei were counterstained with hematoxylin. Subsequently, specimens were dehydrated and mounted with neutral resin and coverslips for histologic analysis. To count the number of labeled cells and avoid counting errors, every tenth section of the eyes was examined with a light microscope (Leica, Germany).

\section{Statistical analysis}

In all quantification procedures, observers were blinded to the nature of the experimental manipulation. All data were analyzed using SPSS version 10.0 and presented in the text as means \pm standard deviations (SD). One-way analysis of variance (ANOVA) was used for comparison of purified rates of the three NSC groups and the results of FQ-PCR, while repeated measurements of ANOVA were used to determine the results of ELISA assay and the numbers of labeled cells by immunohistochemistry in retinas. $P<0.05$ was considered statistically significant.

\section{Results}

\section{The process of NSC purification}

Primary cultures of dissociated subependymal zone tissues of embryonic rats established a population of adherent cells within $24 \mathrm{~h}$ of plating. In the supernatant, floating aggregates (neurospheres) appeared on day 2. NSCs were small and round with little morphological evidence of differentiation and increased in both size and number with time in culture (Figure 1A). We started to passage NSCs while the center of the neurosphere became dark. The self-renewal capacity of these neurospheres was established by plating single cells (refer to Materials and methods) in single wells of 96-well plates. Under these conditions, we observed the proliferation of a single cell into a neurosphere (Figure 1B-1D). Considerably high purity of NSCs was gained by expansive culturing of these clonal neurospheres.

\section{Observation of NSC differentiation process}

The vast majority of cells in the neurospheres obtained by limiting dilution assay expressed nestin immunoreactivity (IR) (Figure 1E). The rates of nestin-positive NSCs of purified groups using limiting dilution assay was higher than that of consecutive passage assay, and both purified groups were higher than that of primary cultured NSCs ${ }^{[15]}$. The differentiated cells cultured with 7 days exposure to $10 \%$ FBS exhib-
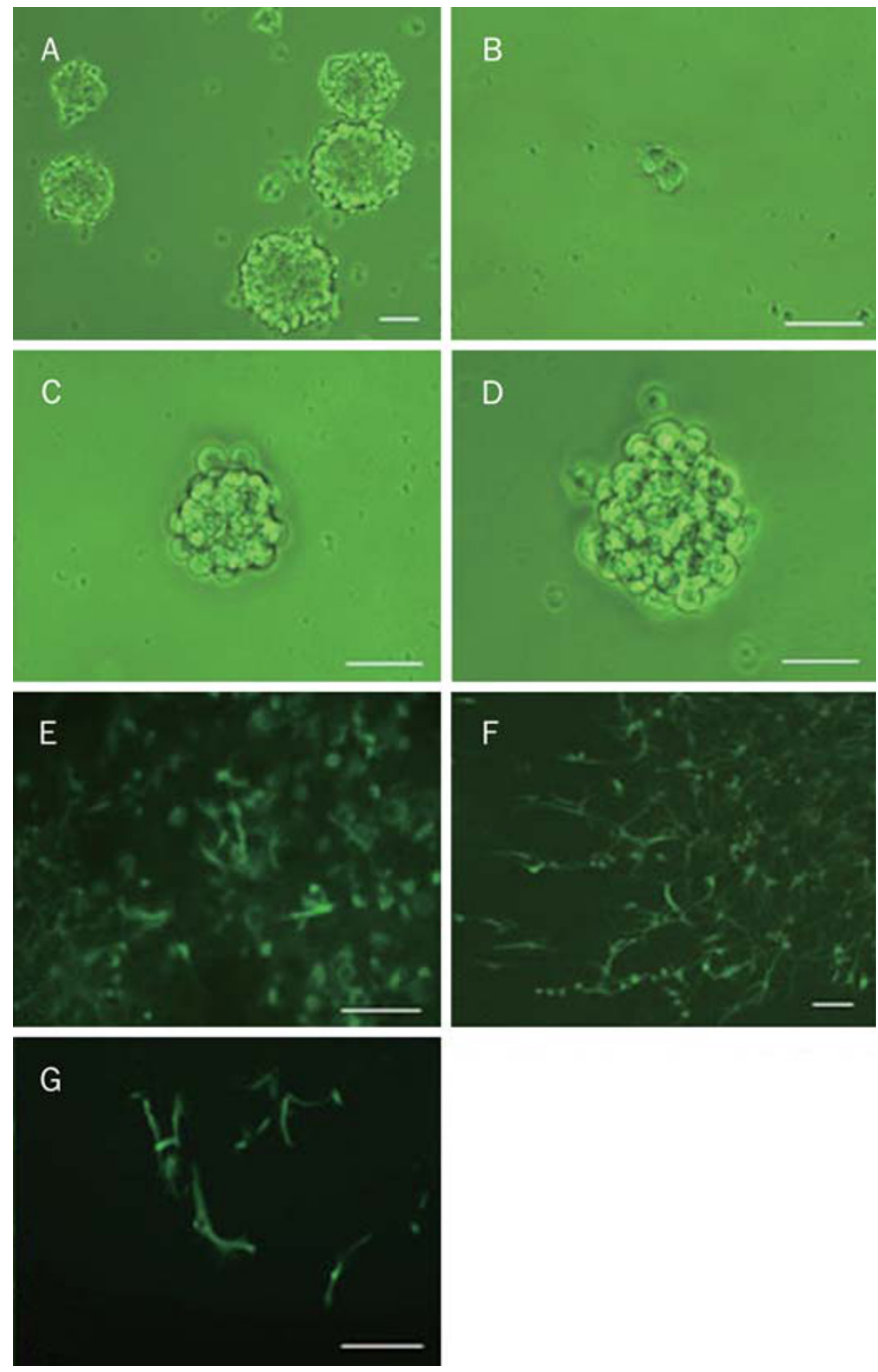

Figure 1. (A) primary neural stem cells. A cell became to divide at d 2 after plating single cells in single wells of 96 -well plates (B) and proliferated continually at d $6(\mathrm{C})$, and neurosphere composed of several decades of cells were formed at day 10 (D). (E-F) Immunocytochemical staining of differentiated neural stem cells (NSCs). (E) nestin; (F) GFAP (glial fibrillary acidic protein); (G) NSE (neurone specific enolase). Bars: $100 \mu \mathrm{m}$.

ited neuronal-type morphology with elliptic cell bodies and bipolar or multipolar extending processes. These cells were immunolabeled for the mature markers GFAP, indicating differentiation along glial lineages, and exhibited similar to neural network connection (Figure 1F), and some were labeled with NSE protein (Figure 1G), indicating differentiation along neuronal lineages.

\section{GFP labeling as an indicator}

As shown in Figure 2A, GFP expression was observed in NSCs $3 \mathrm{~d}$ after infection. Fluorescence intensity of GFP increased gradually in a time-dependent manner (Figure 2B-2C), which suggests that this reporter could be used as an index of detection of transplanted cells in the host in vivo. Consistent with 

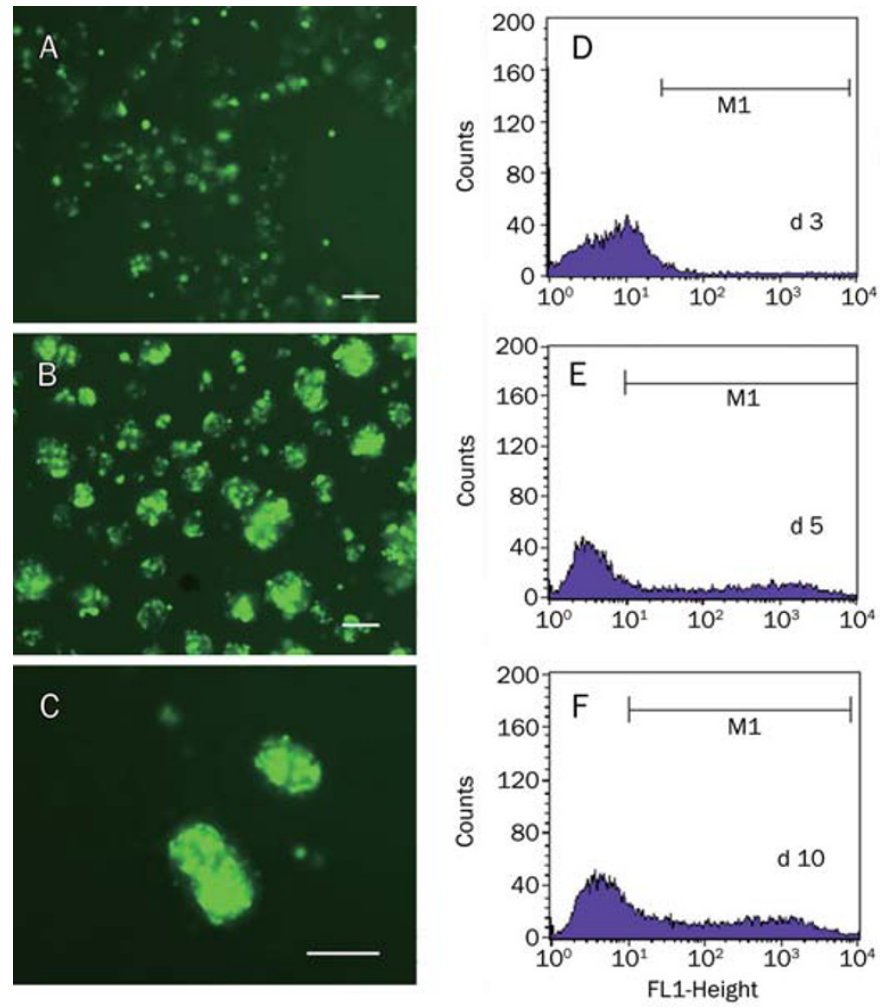

Figure 2. (A-C) GFP expression could be observed in NSCs by fluorescence microscopy at d $3(A)$ and GFP fluorescence intensity became more and more stronger (B-C) with time. Bars: $100 \mu \mathrm{m}$. (D-F) GFP expression rates of NSCs detected by flow cytometry. The M1 pool identifies labeled cells of varying intensity. The pool except M1 identifies unlabaled cells. The ratio of fluorescence cells in M1 gradually increased to almost $47.72 \%$ during the course of the experiment.

results ascribed as above, the results obtained by using flow cytometry also showed that GFP-expressing rates in NSCs exerted a time-dependent fashion (Figure 2D-2F; $6.08 \%$ on day $3,26.57 \%$ on day $5,47.72 \%$ on day 10 ). The high GFP expression level could last for about one month from our observations.

\section{Detecting expression of BDNF using FQ-PCR}

To confirm whether BDNF-NSCs, p-NSCs, and control-NSCs expressed BDNF in cultured NSCs in vitro, mRNA from these NSCs (at the same cell numbers) was amplified using real-time fluorescent quantitative PCR. Using rat-specific primers and probes (refer to Materials and methods), the quantitative neurotrophic factor of BDNF gene expression in vitro was compared with one-way ANOVA among three groups. BDNF-NSCs contained far greater levels of BDNF mRNA $\left[(10.79 \pm 0.34) \times 10^{3}\right.$ copies $\left./ \mu \mathrm{L}, P<0.001\right]$ than $\mathrm{p}-\mathrm{NSCs}$ $\left[(1.45 \pm 0.04) \times 10^{3}\right.$ copies $\left./ \mu \mathrm{L}\right]$ and control-NSCs $\left[(1.83 \pm 0.06) \times 10^{3}\right.$ copies $/ \mu \mathrm{L}]$. BDNF expression in the latter two groups showed no statistical difference $(P>0.05)$, which demonstrated that exogenous gene BDNF had been integrated into the genome of NSCs, and the changes in BDNF expression were not the result of retroviral infection or due to the effect of G418.

\section{ELISA}

The specific ELISA for BDNF protein levels was performed on conditioned media collected from BDNF-NSCs, p-NSCs, and control-NSCs in vitro at different timepoints within $4 \mathrm{~d}$. The statistical significance of the results was evaluated by repeated measurement of ANOVA. BDNF expression displayed a significantly increased tendency with time in the three NSC groups. At day 4, BDNF-NSCs showed a robust release of BDNF with a maximum peak of $824.39 \pm 37.64 \mathrm{ng} / \mathrm{L}$ from total proteins determined by ELISA (Table 1). BDNF protein levels of BDNF-NSCs were highest of the three groups $(P<0.05)$, and there was no statistical difference between the other two groups $(P>0.05$, Table 1$)$, which is consistent with the results obtained by FQ-PCR. These results suggest that engineered NSCs could express exogenous genes and proteins.

The in vivo data suggest that BDNF promotes successful rate of NSC transplantation

An OCT graph of normal rat retina is shown in Figure 3A. OCT examination on BDNF-NSCs-transplanted rats on day 3 demonstrated a large retinal detachment (Figure 3B) at the transplanted site. Only a shallow retinal detachment (Figure 3C) could be seen on days 10-15. The retinal detachment almost completely reattached except for one or two small, occasional blebs (Figure 3D) at one month, and some hyperreflective areas (red/white, presumably corresponding to the donor cells) were observed at the host retinal layers and the RPE. The images of OCT showed that blebs disappeared, and the retinas became flattened, and a hyper-reflective linear

Table 1. ELISA for BDNF (ng/L).

\begin{tabular}{lcccc}
\hline Cell types & 1 day & 2 days & 3 days & 4 days \\
\hline BDNF-NSCs & $95.90(6.65)^{\mathrm{b}}$ & $183.04(7.50)^{\mathrm{b}}$ & $298.69(10.38)^{\mathrm{b}}$ & $824.39(37.64)^{\mathrm{b}}$ \\
p-NSCs & $39.76(5.41)^{\mathrm{d}}$ & $46.59(6.05)^{\mathrm{d}}$ & $50.93(5.37)^{\mathrm{d}}$ & $56.81(5.55)^{\mathrm{d}}$ \\
Control-NSCs & $40.36(6.38)$ & $47.65(3.31)$ & $51.92(6.56)$ & $58.00(5.26)$ \\
\hline
\end{tabular}

Kinetic analysis of BDNF released by BDNF-NSCs and both group of control respectively. Conditioned media were collected after 1, 2, 3, and $4 \mathrm{~d}$ at each time point from three groups. BDNF expression displayed a significant increased tendency with time in all groups, and at the end of $4 \mathrm{~d}$, BDNF protein levels of BDNF-NSCs reached the peak. SD is shown in the brackets. ${ }^{b} P<0.05$ vs $p$-NSCs and control-NSCs, ${ }^{d} P>0.05$ vs control-NSCs [BDNF protein levels of BDNF-NSCs were highest among three groups $(P<0.05)$ and there were no statistical difference between the other two groups $(P>0.05)]$. 

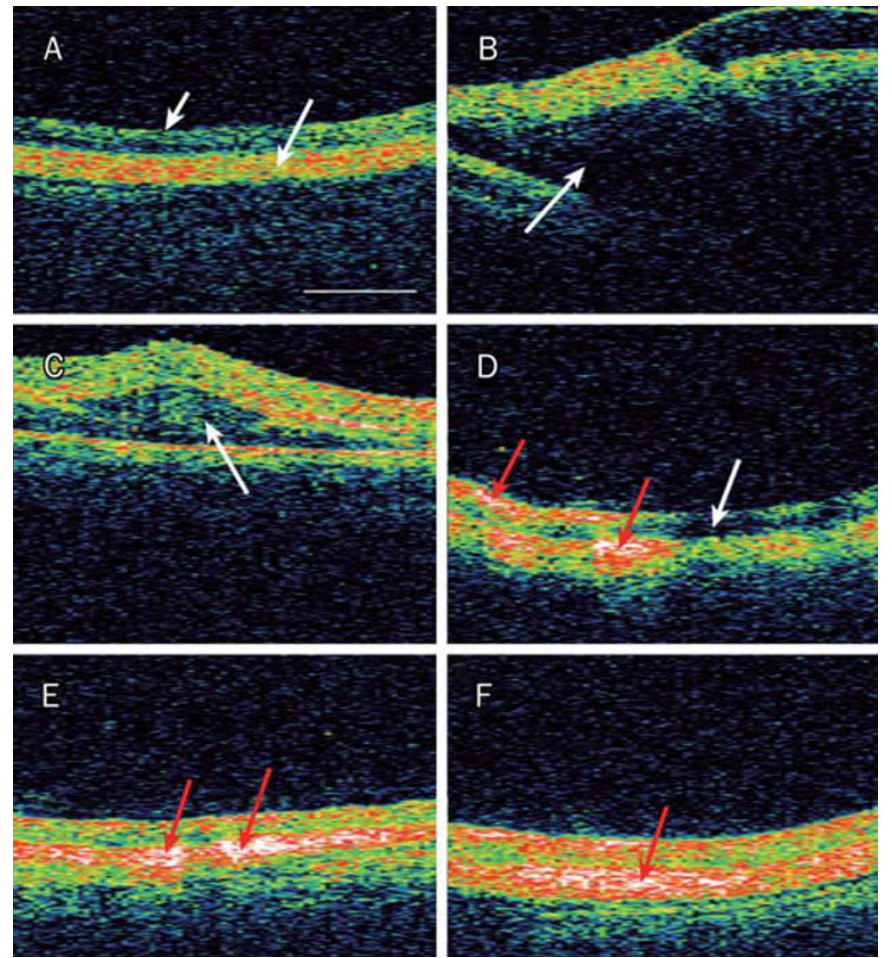

Figure 3. (A) OCT images of normal retina of rat, short arrow: neural retinas layer; long arrow: RPE (retinal pigment epithelium) and Choroid layer. (B-F), OCT examination of BDNF-NSCs transplantation. There is a big retinal detachment $(B$, white arrow) at the injected site at $d 3$. A shallow retinal detachment (C, white arrow) could be seen at d 10-15. The retinal detachment reattached almost except one or two small bleb (D, white arrow) at 1 month and hyper-reflective sites (red/white) probably indicating the graft ( $D$, red arrows) were noted at the host retinal layer and the RPE layer. (E) After 2 month, the bleb almost disappeared and host retina recovered to normal structure. The hyper-reflective bands (red arrow, donor cells) could be easily detected between neural retinas and choroid layers. (F) The intensity and areas of red/white bands (red arrows) became stronger and retinal structure became little vague at 3 months after surgery, which suggested that donor cells perhaps have merged with host retina or undergone proliferation. Bars, $100 \mu \mathrm{m}$.

band (Figure 3E) was formed two months following subretinal transplantation of BDNF-NSCs. This additional hyper-reflective band became larger and likely integrated with host retina or had undergone proliferation at three months (Figure 3F). The same results were observed in the other transplanted cell groups (data not shown). The PBS group showed only retinal detachment, and nothing was observed in the normal control group (data not shown).

\section{HRA examination tracking the migration of transplanted NSCs} GFP-expressing fluorescence of donor cells could be detected by HRA to track the transplanted cells in host tissues. As shown in Figure 4A, $10 \mathrm{~d}$ after BDNF-NSC transplantation, one could observe restricted fluorescence only in the vicinity of the surgical site. It appeared that some fluorescent masses (Figure 4B) migrated slightly from the injection site to the sur-
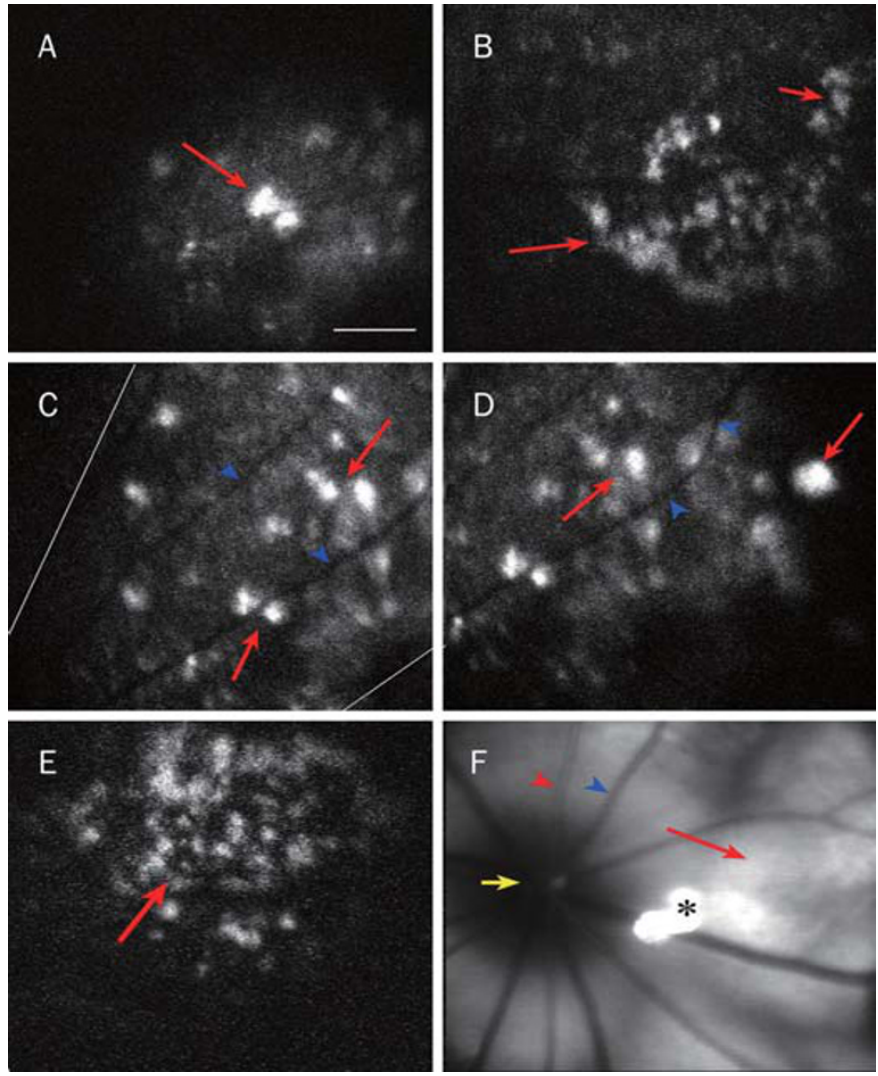

Figure 4. (A-D), HRA of the BDNF-NSCs transplantation at different time points. A little of fluorescence were observed at the surgical site at $d$ 10 (A, red arrow, donor cells). Some fluorescence masses (Figure 4B, short red arrow) migrated slightly from the injected site one month after surgery. Most donor cells migrated to a fan-like shape (Figure 4C) and cell areas and the distance of migration increased at 2 months. Some cells migrated to peripheral retinas at 3 months (Figure 4D). Black lines were retinal vessels of rats (blue arrow heads). The control-NSCs transplant groups displayed a similar phenomenon at the same time point (data not shown), but some cell clumps gathered easily (Figure 4E, red arrow) and the migrating distance were short. The retina of transplanted area was found flat before laser (Figure 4F, yellow arrow: optic nerve head; red arrow head: central retinal arteries; blue arrow head: central retinal veins; red arrow: migrated areas; white point was reflect light). Bars, $200 \mu \mathrm{m}$.

rounding area one month after surgery. Fluorescent areas and intensity became larger and stronger, and the distance of migration increased in a time-dependent manner after transplantation (Figure 4C-4D, 2-3 months), which suggested that cell grafts could not only survive but also proliferate in the host tissue. Most donor cells migrated along the host nerve fiber away from the optic papilla and grew a fan-like shape (Figure 4C). Results from the control-NSCs transplant groups displayed a similar phenomenon at the same time point (data not shown), but some cell clumps were prone to gather (Figure $4 \mathrm{E}$ ), and the migrating distance and cell expanding areas were less than that of the BDNF-NSCs. The retina of transplanted area was found to be flat before laser application (Figure $4 \mathrm{~F}$ ), which was consistent with the results of OCT. PBS control 
and normal control groups showed no fluorescence at any time point (data not shown).

\section{Immunohistochemistry}

HE staining showed retinal structure before surgery (Figure $5 \mathrm{~A})$. Immunohistochemistry of retinas containing transplanted cells confirmed that the nestin-positive donor cells (Figure 5B) existed in the injected site in approximately equal numbers between BDNF-NSCs and control-NSCs. The results showed that there were no cells labeled for NSE or GFAP protein in these two transplanted groups $10 \mathrm{~d}$ after transplantation, which indicated that the injected cells were neural stem cells without differentiation at this time point. One month after transplantation, donor cells occurred both as individual cells and as occasional small clusters of cells attached to the retinal surface. Immunolabeling of retinal sections revealed that a few of the transplanted cells within the recipient tissue expressed NSE (Figure 5C) and GFAP (Figure 5D) and lay in the outer nuclear layer (ONL) and exhibited neuronal-like morphologies with processes, but expression was negative for nestin protein in the retinas. The number of cell immunoreactions with NSE in the BDNF-NSCs group (9.63 \pm 2.37$)$ was slightly higher than that of the control-NSCs group (6.05 \pm 1.08$)$; however, there was no statistical difference among the two groups $(P>0.05)$. The number of GFAP-positive cells was higher in the control-NSCs group. Two months after transplantation, immunohistochemical analysis demonstrated that most of the donor cells appeared to migrate to the deep retina, and few migrated into the inner nuclear layer (INL) of the retina. Within the ONL and outer plexiform layers (OPL) of the retina, we commonly observed bodies and processes of donor cells expressing NSE with extensive branching in the BDNF-NSCs group (Figure 5E). Grafted control-NSC cells remained in the ONL. More NSE-positive cells were found in the BDNF-NSC group $(18.18 \pm 1.55, P<0.05)$ compared to that of the control-NSC group (10.28 \pm 1.32$)$. In contrast, GFAPlabeled cells in the control-NSC group were highest between the two groups. Whole mount analysis indicated that three months after transplantation of donor cells into the subretinal space of normal rats, a large fraction of donor cells expressed the neuronal markers NSE or GFAP. NSE-labeled cell number of BDNF-NSCs (22.87 \pm 3.12$)$ was still higher than the controlNSC group, and a few of the NSE-positive cells had migrated into the inner nuclear layer (INL) of the retina (Figure 5F). The grafts of control-NSCs were still in ONL three months after transplantation (Figure 5G). Occasionally, a few of the grafted cells lay in RPE (Figure 5H) at 3 months.

\section{Discussion}

Our data indicated that pLXSN-BDNF could effectively transfer NSCs. The results from both the in vivo and the in vitro experiments showed that the donor cells could survive, migrate, and differentiate into neuronal and glial cells in the normal adult host retinas, and that BDNF further strengthened this effect. It appears that this method could be used for cell transplantation to cure neuropathy of the eye.
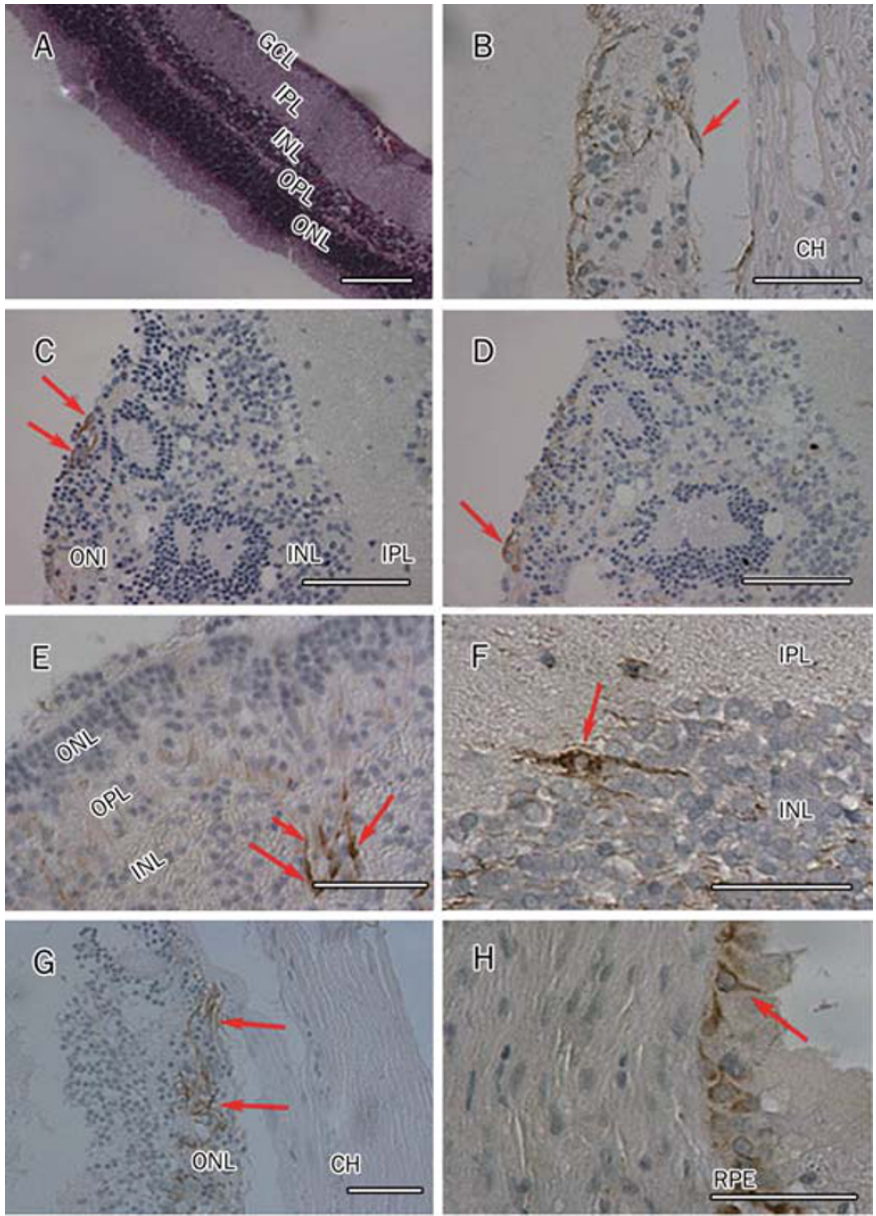

Figure 5. Immunohistochemistry of injected site of rat retinas. HE staining showed normal retinal structure (A). The nestin positive cells of BDNFNSCs group ( $B$, red arrow) existed between the retina and choroid at $d 10$ after transplantation. One month after transplantation, immunolabeling of retinal sections revealed that a few of transplanted cells expressed NSE (C: BDNF-NSCs, red arrows) and GFAP (D, BDNF-NSCs, red arrow) and lay in the outer nuclear layer $(\mathrm{ONL})$ and exhibited neuronal-like morphologies. At 2 months after injected, immonuhischemical analysis demonstrated that most of the donor cells appeared to migrate to the deep retina. Within the $\mathrm{ONL}$ and outer plexiform layers (OPL) of the retina, we commonly observed bodies and processes of donor cells expressing NSE with extensive branching in the BDNF-NSCs group (E, long red arrows: bodies of cells; short red arrow: processes of cells). Whole mount analysis indicated that, 3 months after transplantation of donor cells into the subretinal space of normal rats, a few of NSE positive cells of BDNF-NSCs group had migrated into inner nuclear layer (INL) of the retina (F, red arrow). Graft of controlNSCs were still in ONL at 3 months (G, red arrows). A few of grafted cells lay in RPE ( $\mathrm{H}$, red arrow). BDNF, brain-derived neurotrophic factor; NSCs, neural stem cells; NSE, neurone specific enolase; GFAP, glial fibrillary acidic protein; ONL, outer nuclear layer; INL, inner nuclear layer; OPL, outer plexiform layer; RPE, retinal pigment epithelium. Bars, $100 \mu \mathrm{m}$.

NSCs signal the rapid development of neurology and display vigorous vitality in the regenerative medicine of the nervous system. NSC transplantation has been used by many investigators to observe the effectiveness of curing degenerative diseases and injury reparation of the nervous system because NSCs can not only differentiate to certain cell types, but also 
serve as a vehicle for delivery and expression of the therapeutic genes in the focus of transplantation ${ }^{[16,17]}$. Some hemiparkinsonian model rats that received human tyrosine hydroxylase-1 (HTH-1) gene-transduced donor neural progenitor cells (NPCs) have achieved functional recovery ${ }^{[16]}$. Lu P et al ${ }^{[17]}$ demonstrated that C17.2 neural stem cells can elicit significant host axonal growth after spinal cord injury, presenting a cellular substrate to the lesion site that supports axonal extension even in the absence of stem cell differentiation. The grafting of NSCs to specific regions of the nervous system has previously been reported to induce differentiation of cells to lineages appropriate to the environment where they are placed ${ }^{[18-21]}$. These data strongly support our choice of NSCs as the donor cells for the transplantation because the optic nerve is part of the central nervous system.

Optic neuropathy is a degenerative disease and has no effective treatment in ophthalmology; it is caused mainly by the apoptosis of retinal ganglion cells (RGCs) due to neurotrophin deprivation. Brain-derived neurotrophic factor (BDNF) is a member of the neurotrophic family, and its receptors have been identified on the cells in the RGC layer and inner nuclear layer ${ }^{[22,23]}$. It has been reported that intravitreal injection of BDNF only delayed, but did not rescue, RGC apoptosis in experimental glaucoma rats ${ }^{[24]}$. It is well known that NSCs secret BDNF; however, the endogenous BDNF level is very low and not enough for a therapeutic dose $\mathrm{e}^{[3,4]}$. Also, the transplanted NSCs in the host usually differentiate to glial cells instead of neurons in vivo. Based upon the results obtained from NSC transplantation studies, these investigators concluded that using either NSCs or BDNF alone in the treatment of optic neuropathy is not entirely effective ${ }^{[10,11]}$. Therefore, we hypothesized that the combination of BDNF and NSCs may elevate the treatment effect. In a recent study, NSCs transplanted along with solutions of slow-releasing BDNF into the vitreous cavity of developing rats resulted in the majority of donor cells integrating into the host retina, but the difference was not statistically significant compared with the control group. These data indicated that the neuroprotective effect of BDNF's slow-releasing system on NSCs was limited as well ${ }^{[25]}$. To this end, we planned to utilize gene transfer to ensure continuous and prolonged delivery of neurotrophic factors by the donor cells. In this study, we implemented the overexpression of BDNF in purified NSCs by retroviral vectors with the advantages of low cell toxicity and immune responses, high efficiency and precision of integration, permanent expression of incorporated genes after integration into the chromosome of the host, and no wild virus generation ${ }^{[16,26,27]}$. The BDNFNSCs were labeled with AAV-EGFP and transplanted into the subretinal space of normal rat retinas. The results of HRA and OCT revealed that the donor cells could survive and migrate in the host retina, which are consistent with the results of previous studies ${ }^{[28,29]}$. Three months after transplantation, the immunohistochemical analysis demonstrated that the control-NSCs only distributed at the ONL while BDNFNSCs appeared at the deeper retina of INL, and the graft cells seemed to form synaptic connections with host cells in the retina, which suggested that exogenous BDNF facilitated the migration of transplanted cells. Furthermore, the transplanted BDNF-NSCs differentiated into neurons more efficiently than the control-NSCs in vivo, which was in accordance with the observation that BDNF can help NSCs to differentiate into neurons in vitro ${ }^{[30]}$.

Additionally, there are some advantages in the transplantation process and examinations of our study. Instead of searching for a contact lens to match the small eyeball of rats, a 90D preset lens can introduce a 29-gauge needle into the subretinal space of the host in a complete manner and with only one port, thereby simplifying the operation and reducing tissue damage. To avoid sucking a small amount of vitreous before vitreous cavity injection and the barriers of the posterior vitreous limiting membrane and ILM (internal limiting membrane), we performed subretinal space injection. Our results show that transplanted cells survived well in host retinas without immunorejective reaction, and no obvious inflammation occurred in any recipient rat, but we did not examine the index of immunorejective reaction. The lack of an immunorejective reaction in the recipient rats could be attributed to the following: 1) embryonic NSCs from the rats used for this study may exhibit low immunogenicity, and the BDNF genes are also derived from the rats; 2) we performed the allogeneic graft, and the subretinal space is the immune privilege area in the eyes; 3 ) the rats with reflux and hemorrhage of eyes were excluded from our study, so immunorejection was not observed. This may lead to the success of BDNF-NSC transplantation without any major immunorejection reaction. Thus, the technique of intraocular injection is relatively safe and is easy to assess, and this method could be applied to patients. Nondestructive examination methods (such as HRA and OCT) were employed to track the migration and distribution of transplanted cells in this study, which offered a real-time detection and observation with dynamic and high resolution. These nondestructive examination techniques in vivo accompanied with the relatively easy and safe surgical procedure in our work could lead to more successful transplantation with fewer complications.

In summary, we successfully established a rat model for NSC transplantation with overexpression of BDNF, and our results demonstrated that engineering BDNF into NSCs may elevate the treatment effect of transplantation since BDNF promotes the migration of NSCs and facilitates their neuronic differentiation. However, we do not yet know the ability of genetically modified NSCs to form synapses with other host tissue cells and/or establish the correct neuronal networks to revive architectural and functional reconstruction. These issues need further investigation. Nonetheless, the results of the current study may provide an advanced therapeutic method for optic neuropathy.

\section{Acknowledgments}

This study was supported by a grant from the National Natural Science Foundation of China (№ 30471844).

The authors thank Harbin Veterinary Research Institute (HVRI, China Agricultural Academy of Science, CAAS) for 
providing the experimental location and technical assistance for FQ-PCR.

\section{Author contribution}

Hui-ping YUAN designed the research; Xue-mei ZHOU and Jing-bo SUN performed the research, analyzed the data and wrote the paper; Dong-lai WU provided the experimental location and technical assistance for FQ-PCR; Xin-rong ZHOU, Hong-yi LI, Zheng-bo SHAO contributed to package recombinant retrovirus pLXSN-BDNF; Da-wei SUN contributed to retinal space transplantation surgery and HRA detection. Zhiren ZHANG contributed to revising the paper and helpful discussion.

\section{References}

1 Temple S. Division and differentiation of isolated CNS blast cells in microculture. Nature 1989; 340: 471-3.

2 Cattaneo E, McKay R. Proliferation and differentiation of neuronal stem cells regulated by nerve growth factor. Nature 1990; 347 : 762-5.

3 Gouras P, Du J, Kjeldbye H, Yamamoto S, Zack DJ. Long-term photoreceptor transplants in dystrophic and normal mouse retina. Invest Ophthalmol Vis Sci 1994; 35: 3145-53.

4 Algvere PV, Berglin L, Gouras P, Sheng Y, Kopp ED. Transplantation of RPE in age-related macular degeneration: observations in disciform lesions and dry RPE atrophy. Graefes Arch Clin Exp Ophthalmol 1997; 235: 149-58.

5 Wan J, Zheng H, Chen ZL, Xiao HL, Shen ZJ, Zhou GM. Preferential regeneration of photoreceptor from Müller glia after retinal degeneration in adult rat. Vision Res 2008; 48: 223-34.

6 Ourednik V, Ourednik J, Park KI, Teng YD, Aboody KA, Auguste KI, et al. Neural stem cells are uniquely suited for cell replacement and gene therapy in the CNS. Novartis Found Symp 2000; 231: 242-62; discussion 262-9, 302-6.

7 Flax JD, Aurora S, Yang C, Simonin C, Wills AM, Billinghurst LL, et al. Engraftable human neural stem cells respond to developmental cues, replace neurons, and express foreign genes. Nat Biotechnol 1998; 16: 1033-9.

8 Chacko DM, Rogers JA, Turner JE, Ahmad I. Survival and differentiation of cultured retinal progenitors transplanted in the subretinal space of the rat. Biochem Biophys Res Commun 2000; 268: 842-6.

9 Ahmed S, Reynolds BA, Weiss S. BDNF enhances the differentiation but not the survival of CNS stem cell-derived neuronal precursors. J Neurosci 1995; 15: 5765-78.

10 Kamei N, Tanaka N, Oishi Y, Hamasaki T, Nakanishi K, Sakai N, et al. BDNF, NT-3, and NGF released from transplanted neural progenitor cells promote corticospinal axon growth in organotypic cocultures. Spine 2007; 32: 1272-8.

11 Pluchino S, Quattrini A, Brambilla E, Gritti A, Salani G, Dina G, et al. Injection of adult neurospheres induces recovery in a chronic model of multiple sclerosis. Nature 2003; 422: 688-94.

12 Tropepe V, Sibilia M, Ciruna BG, Rossant J, Wagner EF, van der Kooy D. Distinct neural stem cells proliferate in response to EGF and FGF in the developing mouse telencephalon. Dev Biol 1999; 208: 166-88.

13 Singh SK, Clarke ID, Terasaki M, Bonn VE, Hawkins C, Squire J, et al. Identification of a cancer stem cell in human brain tumors. Cancer
Res 2003; 63: 5821-8.

14 Li HY, Yuan HP, Zhou XR, Shao ZB, Zhou XM. The expression of recombinant retrovirus $\mathrm{pLXSN}$-brain-derived neurotrophic factor in mouse embryonic cell. Chin J Ocular Fundus Dis 2007; 23: 207-8. Chinese.

15 Zhou XM, Yuan HP, Zhou XR, Qu W, Li HY, Shao ZB. Comparison of efficacy of two purified methods for neural stem cells from the subependymal zone of embryonic rat brain. Rec Adv Ophthalmol 2009; 29: 90-3. In Chinese.

16 Kodama Y, Hida H, Jung CG, Baba H, Isono M, Kobayashi $\mathrm{H}$, et al. High titer retroviral gene transduction to neural progenitor cells for establishment of donor cells for neural transplantation to parkinsonian model rats. Neurol Med Chir (Tokyo) 2004; 44: 344-51; discussion 352.

17 Lu P, Jones LL, Snyder EY, Tuszynski MH. Neural stem cells constitutively secrete neurotrophic factors and promote extensive host axonal growth after spinal cord injury. Exp Neurol 2003; 181: 115-29.

18 Brüstle O, Spiro AC, Karram K, Choudhary K, Okabe S, McKay RD. In vitro-generated neural precursors participate in mammalian brain development. Proc Natl Acad Sci USA 1997; 94; 14809-14.

19 Lundberg C, Field PM, Ajayi YO, Raisman G, Björklund A. Conditionally immortalized neural progenitor cell lines integrate and differentiate after grafting to the adult rat striatum. A combined autoradiographic and electron microscopic study. Brain Res 1996; 737: 295-300.

20 Gage FH, Kempermann G, Palmer TD, Peterson DA, Ray J. Multipotent progenitor cells in the adult dentate gyrus. J Neurobiol 1998; 36: 249-66.

21 Suhonen JO, Peterson DA, Ray J, Gage FH. Differentiation of adult hippocampus-derived progenitors into olfactory neurons in vivo. Nature 1996; 383: 624-7.

22 Pease ME, McKinnon SJ, Quigley HA, Kerrigan-Baumrind LA, Zack DJ. Obstructed axonal transport of BDNF and its receptor TrkB in experimental glaucoma. Invest Ophthalmol Vis Sci 2000; 41: 764-74.

23 Perez MT, Caminos E. Expression of brain-derived neurotrophic factor and of its functional receptor in neonatal and adult rat retina. Neurosci Lett 1995; 183: 6-9.

24 Ko ML, Hu DN, Ritch R, Sharma SC, Chen CF. Patterns of retinal ganglion cell survival after brain-derived neurotrophtc factor administration in hypertensive eyes of rats. Neurosci Lett 2001; 305; 139 42.

25 Suzuki T, Ooto S, Akagi T, Amemiya K, Igarashi R, Mizushima Y, et al. Effects of prolonged delivery of brain-derived neurotrophic factor on the fate of neural stem cells transplanted into the developing rat retina. Biochem Biophys Res Commun 2003; 309: 843-7.

26 Bushman FD. Retroviral integration and human gene therapy. J Clin Invest 2007; 117: 2083-6.

27 Miller AD, Buttimore C. Redesign of retrovirus packaging cell lines to avoid recombination leading to helper virus production. Mol Cell Biol 1986; 6: 2895-902.

28 Qiu G, Seiler MJ, Mui C, Arai S, Aramant RB, de Juan E Jr, et al. Photoreceptor differentiation and integration of retinal progenitor cells transplanted into transgenic rats. Exp Eye Res 2005; 80: 515-25.

29 Cong L, Sun D, Zhang Z, Jiao W, Rizzolo U, Peng S. A novel rabbit model for studying RPE transplantation. Invest Ophthalmol Vis Sci 2008; 49: 4115-25.

30 Choi KC, Yoo DS, Cho KS, Huh PW, Kim DS, Park CK. Effect of single growth factor and growth factor combinations on differentiation of neural stem cells. J Korean Neurosurg Soc 2008; 44: 375-81. 